\title{
Ox-low density lipoprotein (ox-LDL) increased the susceptibility of primary nephrotic syndrome (PNS) by regulating the Treg/Th17 ratio via the MEG3 signaling
}

Type

Research paper

\section{Keywords}

FOXP3, RORyt, MEG3, Treg/Th17, miR-125a, primary nephrotic syndrome, ox-low density lipoprotein, miR-17

\begin{abstract}
Introduction

Patients with primary nephrotic syndrome (PNS) were reported to exhibit the evident imbalance between the number of Th17 and Treg cells in their peripheral blood monocytes (PBMCs), which might be the immunological basis of the disease.

Material and methods

40 PNS patients and 42 healthy individuals were recruited in this study. FCM assay was used to observe the levels of Treg and Th17 cells.
\end{abstract}

Results

The Treg/Th17 ratio was evidently decreased in PNS patients. The levels of MEG3 and RORyt were increased in the PNS group, while the levels of miR-17, miR-125a and FOXP3 mRNA were reduced in the PNS group. Moreover, the levels of IL- 6 and IL- $1 \beta$ were highly increased in PNS patients. Oxlow density lipoprotein (ox-LDL) treatment significantly increased the levels of MEG3 and RORyt $\mathrm{mRNA} /$ protein while decreasing the levels of miR-17, miR-125a, and FOXP3 mRNA/protein in THP-1 cells, and the transfection of MEG3 siRNA partly alleviated the dysregulation in the expression of MEG3, relevant miRNAs and relevant mRNAs induced by ox-LDL. Also, the expression of miR-17 and miR-125a was evidently decreased upon the successful transfection of MEG3, but the RORYt $\mathrm{mRNA} /$ protein levels were promoted while the FOXP3 mRNA/protein levels were inhibited.

\section{Conclusions}

Our results demonstrated that ox-LDL could promote the inflammatory response of PNS by decreasing the Treg/Th17 ratio via activating the MEG3 signaling. The activation of MEG3/miR-125a/FOXP3 axis and MEG3/miR-17/RORyt axis respectively lead to the Treg/Th17 imbalance, which resulted in up-regulated IL- 6 and IL-1 $\beta$ levels, thus increasing the susceptibility of PNS. 

(PNS) by regulating the Treg/Th17 ratio via the MEG3 signaling

1. Department of Nephrology, Kidney Hospital, The First Affiliated Hospital of Xi'an Jiaotong University, Xi'an710061, P.R. China

${ }^{*}$ Correspondence to: Shifeng Yang, Department of Nephrology, Kidney Hospital, The First Affiliated Hospital of Xi'an Jiaotong University, 277 West Yanta Road, Xi'an, Shaanxi Province, People's Republic of China

$9 \quad$ Email: orthomedical@yeah.net

Tel: 0086-29-85323951

\section{Abstract}

Background: Patients with primary nephrotic syndrome (PNS) were reported to exhibit the evident imbalance between the number of Th17 and Treg cells in their peripheral blood monocytes (PBMCs), which might be the immunological basis of the disease. Method: 40 PNS patients and 42 healthy individuals were recruited in this study. FCM assay was used to observe the levels of Treg and Th17 cells. Result: The Treg/Th17 ratio was evidently decreased in PNS patients. The levels of MEG3 and RORyt were increased in the PNS group, while the levels of miR17, miR-125a and FOXP3 mRNA were reduced in the PNS group. Moreover, the levels of IL-6 and IL-1 $\beta$ were highly increased in PNS patients. Ox-low density lipoprotein (ox-LDL) treatment significantly increased the levels of MEG3 and RORyt mRNA/protein while decreasing the levels of miR-17, miR-125a, and FOXP3 mRNA/protein in THP-1 cells, and the transfection of MEG3 siRNA partly alleviated the dysregulation in the expression of MEG3, relevant miRNAs and relevant mRNAs induced by ox-LDL. Also, the expression of miR-17 and miR-125a was evidently decreased upon the successful transfection of MEG3, but the RORyt mRNA/protein levels were promoted while the FOXP3 mRNA/protein levels were inhibited. Conclusion: Our results demonstrated that ox-LDL could promote the inflammatory response of PNS by decreasing the Treg/Th17 ratio via activating the MEG3 signaling. The activation of MEG3/miR-125a/FOXP3 axis 
and MEG3/miR-17/RORyt axis respectively lead to the Treg/Th17 imbalance, which resulted in up-regulated IL-6 and IL-1 $\beta$ levels, thus increasing the susceptibility of PNS.

\section{Abbreviation list}

HC: healthy control

PNS: primary nephrotic syndrome

PBMC: peripheral blood monocyte

MCNS: minimal change nephritic syndrome;

MN: membranous nephropathy;

MsPGN: mesangial proliferative glomerulonephritis;

FSGS: focal segmental glomerulosclerosis

Running title: ox-LDL affects MEG3 signaling in primary nephrotic syndrome

Key words: primary nephrotic syndrome, ox-low density lipoprotein, Treg/Th17, MEG3, FOXP3, miR-125a, miR-17, RORyt

\section{Introduction}

As one of the most widespread renal ailment in child patients, primary nephrotic syndrome (PNS) has been deemed as a major cause of chronic renal failures among Chinese children [1]. The features of PNS include elevated permeability of the barrier of glomerular filtration and resulting oedema, hypoalbuminaemia as well as proteinuria. So far, the mechanism underlying the PNS pathogenesis is still mostly unknown. Some scholars assume that the PNS is actually caused by the deregulation of T cells or the abnormality in T cell responses [2-4]. It was also suggested that the imbalance between the number of Th1 and Th2 cells as well as the imbalance between the number of Th17 and Treg cells lead to PNS $[4,5]$. It was additionally found that ox-LDL plays a stronger role in reducing the count of Treg cells than in reducing the count of Th17 cells, and the discrepancy between the count of Th17 and Treg cells might be responsible for inducing the pathogenesis of the PNS condition in children. Th17 as well as Treg cells, both of which are 
differentiated from naïve CD4+ T-cells, participate in the control of inflammatory responses. In particular, the effector T-cells drive the immunity while promoting inflammation, and the inhibitory Treg cells exert an opposing effect by reducing the activity of effector T-cells while reducing the level of inflammatory responses [6]. The equilibrium between the number of immuno-suppressive Foxp3+ Treg cells and the number of pro-inflammatory Th17 cells exemplifies a critical factor in regulating the homeostasis of the immune system. Furthermore, a decline in the number of Treg cells can cause immune reactions dominated by Th2 cells, resulting in their increased infiltration into eosinophilic tissues [7]. In addition, Th17 and IL -17 were shown to play an essential role in the PNS pathogenesis in children by lowering the expression of podocalyxin as well as by causing the apoptosis of podocytes [4].

LncRNAs are associated with the progression of many diseases, such as asthma, via posttranscriptional or transcriptional regulation of protein expression by means of various mechanisms $[8,9]$. Some of such mechanisms could be explained via the function of competing endogenous RNAs (ceRNAs), i.e., IncRNAs may act as ceRNAs to competitively bind to certain miRNAs via base pair complementation, thus reducing the binding of such microRNAs to target genes, so as lead directly to the changes in target gene expression [10]. It was found that the abnormalities in IncRNA-MEG3 in CD4 + T cells could regulate the Treg/Th17 imbalance in asthma patients by functioning as a competing endogenous RNA of microRNA-17/ RORyt [11]. Also, it was shown that the highly-expressed MEG3 could directly suppress expression of miR-125a-5p. Alternatively, it was likewise noticed that the overexpression of miR-125a-5p triggered the rise of Foxp3 expression as well as the decrease in RORgt expression in the CD4+ cells isolated from ITP patients, indicating that miR-125a-5p actually participated in the pathogenesis of ITP partially via the regulation of Tregs as well as also Th17 cells [12].

It has been reported that patients with PNS exhibited the evident imbalance between the number of Th17 and Treg cells in their PBMCs, which might be the immunological basis of the disease [13]. The ox-low density lipoprotein (ox-LDL) was proved to alter the dynamic profile of Th17 and Treg cells $[14,15]$. The MEG3/miRNA-17/RORyt axis and MEG3/miR-125a-5p axis were involved in asthma and immune thrombocytopenic purpura, respectively [11, 12]. In this study, we hypothesized that the activation of MEG3 signaling could influence the imbalance of 
Th17/Treg cells in PNS patients. And the administration of ox-LDL might influence the susceptibility of PNS by influencing the expression of MEG3 and its subsequent target genes and proteins. Therefore, we recruited PNS patients to study the effect of ox-LDL on the imbalance of Th17/Treg cells. We also performed Western blot, RT-qPCR and ELISA assays to study the molecular mechanisms underlying the role of ox-LDL in the pathogenesis of PNS.

\section{Materials and Methods}

\section{Patient recruitment and patient characteristics}

In this study, 40 PNS patients were recruited as the PNS group, while 42 healthy individuals were recruited as the healthy control (HC) group. Peripheral blood samples as well as demographic and clinical characteristics were collected from all patients in both groups, and then compared using the Student's t tests. In addition, Th17 cells were isolated from the peripheral blood samples and then assayed for target gene expression. Institutional ethical committee has approved the protocol of this study.

\section{Assay of Th17 cells by utilizing flow cytometry}

$5 \mathrm{~mL}$ of peripheral venous blood samples were collected from each of the subjects in the two groups under fasting conditions. In the next step, the samples were centrifugally to isolate $3 \mathrm{x}$ $10^{9} / \mathrm{L}$ of mononuclear cells from each sample. Then, roughly $2 \mathrm{~mL}$ of each blood sample were moved into a 24-well cell culture plate and then added with $1.0 \mu \mathrm{M}$ per well of Monensin, 60 $\mu \mathrm{g} / \mathrm{L}$ per well of Buddha wave ester, as well as $100 \mu \mathrm{M}$ per well of streptin. Subsequently, the plate was incubated in a $5 \% \mathrm{CO} 2$ incubator for $5 \mathrm{~h}$ at $37^{\circ} \mathrm{C}$ before the cell suspension was moved into a sterile $2 \mathrm{~mL}$ centrifuge tube, which was then centrifuged at for 6 minutes at $3000 \mathrm{rpm}$ to collect the cell pellet. In the next step, the cell pellet was cleaned with PBS to get rid of the residue liquid before $15 \mu \mathrm{L}$ of CD4 as well as $15 \mu \mathrm{L}$ of IL17a antibodies were added into each tube. After 30 minutes of incubation in the dark, the cells were fixed in a fixation buffer at $5^{\circ} \mathrm{C}$ for $10 \mathrm{~min}$ in the dark, centrifuged, permeabilized in $1 \mathrm{~mL}$ of permeabilization buffer, rinsed with PBS, and respectively stained using PE-tagged anti-IL-17 antibodies as well as PE-tagged anti-IgG1 antibodies for flow cytometry. 


\section{Assay of Treg cells by utilizing flow cytometry}

110

111

112

113

114

115

116

117

118

119

120

121

122

123

124

125

126

127

128

129

130

131

132

133

134

135

$2 \mathrm{~mL}$ of peripheral venous blood samples were collected from each of the subjects in the two groups under fasting conditions. In the next step, each sample was stained using $15 \mu \mathrm{L}$ of CD4 as well as $15 \mu \mathrm{L}$ of CD25 antibodies for 30 minutes of incubation in the dark before the cells were fixed in $50 \mu \mathrm{L} /$ tube of the fixation buffer at $5^{\circ} \mathrm{C}$ for $10 \mathrm{~min}$ in the dark, centrifuged, permeabilized in $1 \mathrm{~mL}$ of permeabilization buffer, rinsed with PBS, and respectively stained using PE-tagged antiFoxp3 antibodies as well as PE-tagged anti-IgG1 antibodies for flow cytometry.

\section{Evaluation of the Th17/Treg population in total peripheral blood mononuclear cells}

$5 \mathrm{~mL}$ of peripheral venous blood samples were collected from each of the subjects in the two groups under fasting conditions. In the next step, the samples were centrifugally to isolate mononuclear cells, which were seeded into a 24 -well cell culture plate at $1 \times 10^{6}$ cells per well. In the next step, the cells were randomly divided into four groups, i.e., a blank group of mononuclear cells treated with ox-LDL at a $0.0 \mu \mathrm{g} / \mathrm{mL}$ concentration, a $0.1 \mu \mathrm{g} / \mathrm{mL}$ ox-LDL group of mononuclear cells treated with ox-LDL at a concentration of $0.1 \mu \mathrm{g} / \mathrm{mL}$, a $1.0 \mu \mathrm{g} / \mathrm{mL} \mathrm{ox}-\mathrm{LDL}$ group of mononuclear cells treated with ox-LDL at a concentration of $1.0 \mu \mathrm{g} / \mathrm{mL}$, and a 10.0 $\mu \mathrm{g} / \mathrm{mL}$ ox-LDL group of mononuclear cells treated with ox-LDL at a concentration of $10.0 \mu \mathrm{g} / \mathrm{mL}$. Subsequently, the plate was incubated in a $5 \% \mathrm{CO} 2$ incubator for $24 \mathrm{~h}$ at $37^{\circ} \mathrm{C}$ and the cells were collected for flow cytometry to determine the number of Th17 and Treg cells in each sample.

\section{Cell culture}

THP-1 cells were acquired from ATCC and then cultured in a DMEM medium containing $10 \%$ of fetal bovine serum and appropriate penicillin and streptomycin antibiotics (Gibco, Thermo Fisher Scientific, Waltham, MA). The cell culture conditions were at $37^{\circ} \mathrm{C}$ and $5 \% \mathrm{CO}_{2}$ in a fully humidified incubator. After reaching $80 \%$ confluence, the cells were divided into several cellular models. In cell model 1, the THP-1 cells were divided into 3 groups, i.e., 1. PBS group (THP-1 cells treated with PBS only); 2. ox-LDL + NC siRNA group (THP-1 cells treated with ox-LDL and then transfected with a NC siRNA); and 3. ox-LDL+ MEG3 siRNA group (THP-1 cells treated with ox-LDL and then transfected with MEG3 siRNA). In cell model 2, the THP-1 cells were divided into 2 
groups, i.e., 1. NC group (THP-1 cells transfected with a NC plasmid); and 2. p-MEG3 group (THP1 cells transfected with the p-MEG3 plasmid). All cell treatment and transfection lasted $48 \mathrm{~h}$, and the transfection was carried out using Lipofectamine 2000 (Invitrogen, Carlsbad, CA) in accordance with the protocol provided by the reagent manufacturer. After $48 \mathrm{~h}$ of transfection, the transfected cells were harvested to assay the expression of target genes.

\section{Real time PCR}

The collected cell samples were processed by using a Trizol kit (Ambion, Foster City, CA) in accordance with the protocol provided by the assay kit manufacturer to isolate the total RNA content. In the next step, the isolated RNA pellet was resuspended in RNase free water and subject to reverse transcription to generate cDNA. In particular, the synthesis of cDNA using isolated miRNA samples was carried out by using a miScript II RT assay kit (Qiagen, Hilden, Germany) in accordance with the protocol provided by the assay kit manufacturer. On the other hand, the synthesis of cDNA using isolated mRNA samples was carried out by using a GScript FirstStrand cDNA Synthesis kit (GeneDirex, New Taipei, Taiwan) in accordance with the protocol provided by the kit manufacturer. In the next step, the expression of MEG3, relevant miRNAs and relevant mRNAs in each sample was determined by real time PCR, which was carried out on a Light Cycler 480 real-time PCR machine (Roche, Basel, Switzerland) by using a miScript SYBR Green PCR assay kit (Qiagen, Germantown, MD) in accordance with the protocol provided by the assay kit manufacturer. Finally, the relative expression of MEG3, relevant miRNAs and relevant mRNAs in each sample was determined by using the $\Delta \Delta \mathrm{Ct}$ approach [16].

\section{Vector construction, mutagenesis and dual luciferase assay}

Our preliminary bioinformatic analysis predicted a putative binding site of miR-17 on MEG3, as well as a miR-17 putative binding site in the 3'UTR of RORyt mRNA. Therefore, subsequent luciferase assays were carried out to validate regulatory relationship between miR-17 and MEG3 as well as between miR-17 and RORyt mRNA. In brief, wild type sequences of MEG3 as well as RORyt mRNA containing the miR-17 putative binding sites were inserted into pcDNA (Promega, Madison, WI) luciferase reporting vectors, respectively, to generate wild type vectors of MEG3 as well as RORyt mRNA, respectively. At the same time, site-directed mutagenesis was carried 
out by using a Quick Change Mutagenesis assay kit (Stratagene, San Diego, CA) in accordance with the protocol provided by the assay kit manufacturer to generate site-directed mutations in the miR-17 putative binding sites on MEG3 as well as RORyt mRNA, respectively, and the mutant sequences of MEG3 as well as RORyt mRNA containing the miR-17 putative binding sites were also inserted into pcDNA luciferase reporting vectors, respectively, to generate mutant type vectors of MEG3 as well as RORyt mRNA. In the next step, THP-1 cells were transfected with wild type/mutant sequences of MEG3 or RORyt mRNA in conjunction with miR-17 mimics or a scramble control using Lipofectamine 2000 (Invitrogen, Carlsbad, CA) in accordance with the protocol provided by the reagent manufacturer. After $48 \mathrm{~h}$ of transfection, the luciferase activity of transfected cells was measured by using a Dual-Luciferase assay kit (Promega, Madison, WI) in accordance with the protocol provided by the assay kit manufacturer on a Lumat 9507 LB (Berthold Technologies, Oak Ridge, TN) luminometer.

\section{Cell proliferation}

The proliferation status of cell samples was measured by using a xCELLigence RTCA DP assay kit (ACEA Biosciences, San Diego, CA) in accordance with the protocol provided by the assay kit manufacturer.

\section{Statistical analysis}

All data were expressed as mean $\pm \mathrm{SD}$, and all statistical analyses were carried out by utilizing $\mathrm{R}$ Statistical Software. A p-value of lower than 0.05 was considered to be statistically significant. All inter group comparisons were done by using Student's t tests.

\section{Results}

\section{Patient recruitment and patient characteristics}

In this study, 40 PNS patients were recruited into the PNS group, while 42 healthy individuals were recruited into the healthy control $(\mathrm{HC})$ group. The demographic and clinical characteristics were collected and recorded in Table 1. No obvious differences were found between the PNS group and the $\mathrm{HC}$ group. 
The FCM analysis was performed to observe the percentage of Th17 and Treg cells in the patient groups. As shown in Fig.1, the percentage of Th17 cells in the PNS group was significantly increased compared with that in the HC group (Fig.1A), whereas the percentage of Treg cells in the PNS group was markedly reduced (Fig.1B).

Expression of MEG3, miR-17, miR-125a, RORyt mRNA and FOXP3 mRNA in PNS and HC groups

RT-qPCR results of PMBCs collected from the patient groups showed an increased MEG3 level (Fig.2A) in the PNS group, while the expression of miRNAs including miR-17 (Fig.2B) and miR-125a (Fig.2D) was significantly reduced in patients with PNS. The expression of mRNAs including RORyt mRNA and FOXP3 mRNA showed a different tendency. Compared with the HC group, the PNS group showed an increased RORyt mRNA level (Fig.2C) and reduced FOXP3 mRNA level (Fig.2E). Moreover, the plasma level of MEG3 (Fig.3A) was also increased while the plasma levels of miR17 (Fig.3B) and miR-125a (Fig.3C) were both decreased in PNS patients.

\section{Levels of IL-6 and IL-1 $\beta$ in PNS and HC groups}

ELISA assay was conducted to observe the levels of pro-inflammatory cytokines including IL-6 and IL-1 $\beta$ in the patient groups. Accordingly, both the levels of IL-6 (Fig.4A) and IL-1 $\beta$ (Fig.4B) were highly increased in PNS patients.

\section{Ox-LDL participated in the signaling of MEG3}

208 In this study, three groups of THP-1 cells were set up: 1. PBS group in which the cells were treated with PBS only; 2. Ox-LDL+NC siRNA group in which the cells transfected with NC siRNA were treated with ox-LDL; 3. Ox-LDL+MEG3 siRNA group in which the cells transfected with MEG3

211 siRNA were treated with ox-LDL. RT-qPCR results indicated that, compared with the PBS group, 212 the expression levels of MEG3 (Fig.5A) and RORyt mRNA (Fig.5C) and protein (Fig.5F) were 213 evidently higher while the expression levels of miR-17 (Fig.5B), miR-125a (Fig.5D), and FOXP3 214 mRNA (Fig.5E) and protein (Fig.5G) were markedly reduced by the ox-LDL treatment of NC siRNA215 transfected THP-1 cells. Moreover, the transfection of MEG3 siRNA not only reduced the 
expression of MEG3 up-regulated by ox-LDL, but also partly recovered the dysregulation of

217 relevant miRNAs and mRNAs induced by ox-LDL.

218

219

220

221

222

223

224

225

226

227

228

229

230

231

232

233

234

235

236

237

238

239

240

241

242

\section{The signaling of MEG3}

THP-1 cells were transfected with plasmids carrying MEG3. Then, the expression levels of MEG3, relevant miRNAs, relevant mRNAs and relevant proteins were observed. Accordingly, upon the successful transfection of MEG3 (Fig.6A), the expression of miRNAs including miR-17 (Fig.6B) and miR-125a (Fig.6D) was evidently decreased, while the expression of RORyt mRNA (Fig.6C) and protein (Fig.6F) was promoted. The expression of FOXP3 mRNA (Fig.6E) and protein (Fig.6G) was also inhibited in the presence of MEG3.

\section{Establishment of the MEG3 axis}

As shown in Fig.7A, bioinformatic tools predicted a putative binding site of miR-17 on MEG3, and the subsequent luciferase assay validated that the relative luciferase activity was only reduced in THP-1 cells co-transfected with MEG3 and miR-18. Also, a miR-17 putative binding site was observed in the 3'UTR of RORyt mRNA (Fig.7B), and the luciferase assay also validated that RORyt mRNA was targeted by miR-17, thus establishing the MEG3/miR-17/RORyt axis. As shown in Fig.7C, miR-125a could bind to MEG3, and the transfection of miR-125a also reduced the luciferase activity of MEG3 in THP-1 cells, validating the signaling pathway of MEG3/miR125a/FOXP3.

\section{Discussion}

In this study, we enrolled subjects with or without PNS and compared their expression of candidate IncRNAs, miRNAs, and target genes. We found that, in the PNS group, the percentage of Th17 cells was increased whereas the percentage of Treg cells was reduced. In the PBMC and plasma samples collected from the PNS group, an increased MEG3 level was presented with reduced levels of miR-17 and miR-125a. The mRNA level of RORyt was increased while the FOXP3 mRNA level was reduced in the PNS group. Moreover, the levels of pro-inflammatory cytokines including IL-6 and IL-1 $\beta$ were highly increased in PNS patients. A past study revealed that ox-LDL induced a stronger effect on Treg cells as compared to the effect on Th17 cells in children PNS 
243 [14]. It was additionally disclosed that ox-LDL reduced the viability as well as proliferation of cells 244 to promote persistent inflammation as well as apoptosis. It was also disclosed that ox-LDL plays 245 its role through upregulating the expression of MEG3 in Raw264.7 cells [17]. Many studies showed that three cytokines play important roles in coronary heart diseases. For example, IL-5

247 links natural and adaptive immunity to the epitopes of low-density lipoprotein (LDL) that has been oxidized to induce the growth of cells carrying IgM antibodies that are specific to oxLDL.

249 Furthermore, IL-10 as well as TGF-B are both related to Treg cells by playing critical and non250 redundant roles in preventing the formation of atherosclerosis by means of their 251 immunosuppressive, anti-inflammatory, as well as vasculo-protective features [18]. Naive CD4+ 252 T cells in mice activated by TGF- $\beta$ as well as IL-6 result in the activation of retinoic acid-related 253 orphan nuclear receptor $y t$ (ROR $p t$ ), which controls the differentiation of Th17 cells [19]. It was 254 also discovered that Th17 cells results in pediatric PNS by elevating the expression of IL-23, IL-1 $\beta$, 255 IL-6, as well as RORc [4].

256 Past research has shown that IncRNA-MEG3 regulates the balance between Treg and Th17 cells 257 [12]. For example, qRT-PCR showed the positive IncRNA-MEG3 as well as microRNA-17 258 expression in the CD4 + T cells isolated from the peripheral blood samples of patients with asthma, 259 while the increased expression of IncRNA MEG3 was accompanied by the increased expression 260 of microRNA-17, suggesting that the IncRNA MEG3 may disrupt the equilibrium between Treg 261 and Th17 cells in the patients with asthma [11]. Bioinformatic analysis also illustrated the 262 presence of 4 binding sites between miR-125a-5p and MEG3, and MEG3 inhibited the expression 263 of miR-125a-5p by interacting with it directly, so as to induce the imbalance between the number 264 of Treg and Th17 cells in ITP [12]. Another research also revealed that IncRNA MEG3 can work as 265 a ceRNA to suppress the expression of microRNA-17 while upregulating the expression of RORpt, 266 causing the hyperfunction of Th17 as well as a reduced Treg/Th17 ratio [11]. In this study, we 267 also performed bioinformatic and luciferase assays to validate that MEG3 can sponge miR-17 and 268 miR-125a expression, and that RORyt mRNA was targeted by miR-17, thus establishing the 269 MEG3/miR-125a/FOXP3 axis and MEG3/miR-17/RORpt axis.

270 Interesting, the depletion in the number of Foxp3+CD4+ Treg cells causes severe auto-immunity, 271 immuno-pathology, as well as allergy [20]. It was also shown that the number of Treg cells was 
272 significantly reduced in PNS patients, suggesting that the Treg cells play a key protective role in 273 the onset of proteinuria as well as PNS [21, 22]. It was also shown that the effect of IncRNA MEG3 274 on targeting microRNA-17 and RORpt affects the activity of Th17 cells. Thus, the abnormalities in 275 the expression of IncRNAs as well as microRNAs in CD4 + T cells can help to diagnose asthma [11]. 276 In this study, we found that the expression levels of MEG3 and RORyt mRNA/protein were 277 evidently higher while the expression levels of miR-17, miR-125a, and FOXP3 mRNA/protein were 278 markedly reduced by the ox-LDL treatment of NC siRNA-transfected THP-1 cells, and the transfection of MEG3 siRNA partly recovered the dysregulation in the expression of MEG3, relevant miRNAs and relevant mRNAs induced by ox-LDL. Also, the expression of miRNAs including miR-17 and miR-125a was evidently decreased upon the successful transfection of MEG3, which also promoted the RORyt mRNA/protein expression while inhibiting FOXP3 mRNA/protein expression.

284

RORyt has been considered as a crucial transcription factor in Th17 cells. Certainly, the RORyt expression in naïve CD4+ T cells induced by retroviral vectors promoted their differentiation to cells with IL-17A as well as IL-17F expression $[19,23]$. Given that RORyt is crucial for the polarization of Th17 cells in mice, cytokines IL-6 as well as also TGF $\beta$ can increase RORyt expression in Th17 cells [19]. Interestingly, mice fed with a diet enriched in trans fats revealed greater IL-17 as well as RORyt expression along with elevated Th17 responses in colitis and severe intestinal inflammation [24, 25].

\section{Conclusion}

Our results demonstrated that ox-LDL could promote the inflammatory responses of PNS by upregulating the expression levels of $\mathrm{L}-6$ and $\mathrm{IL}-1 \beta$. The MEG3/miR-125a/FOXP3 aixs and MEG3/miR-17/RORyt axis were established by our study, indicating the up-regulated MEG3 expression induced by administration of ox-LDL could respectively down-regulate the expression of FOXP3 and up-regulate the expression of RORyt. Accordingly, the dysregulation of FOXP3 and RORyt respectively led to the decreased Treg/Th17 ratio, thus resulting in the up-regulation of IL-6 and IL-1 $\beta$ in PNS.

\section{Conflict of interest}


None

301

302 303

3121 Plumb L, Boother EJ, Caskey FJ, Sinha MD, Ben-Shlomo Y: The incidence of and risk factors

\section{Acknowledgement}

This work was supported by the Basic Research Plan of Natural Science of Shaanxi Province (2019JM-537).

\section{Author contributions}

$\amalg$, XPW and XFX performed the majority of the laboratory work, statistics and data analysis and participated in writing. PL and SFY were involved in writing. All authors conceived and designed the experiments and drafted the manuscript.

\section{Data availability statement}

The data sets generated during and/or analyzed during the current study are available from the corresponding author on reasonable request.

\section{References} for late presentation of childhood chronic kidney disease: A systematic review and metaanalysis. PLoS One 2020;15(12):e0244709.

2 Sellier-Leclerc AL, Duval A, Riveron S, Macher MA, Deschenes G, Loirat C, Verpont MC, Peuchmaur M, Ronco P, Monteiro RC, Haddad E: A humanized mouse model of idiopathic nephrotic syndrome suggests a pathogenic role for immature cells. J Am Soc Nephrol 2007;18:2732-2739.

3 Shalhoub RJ: Pathogenesis of lipoid nephrosis: a disorder of T-cell function. Lancet 1974;2:556-560.

4 Wang L, Li Q, Wang L, Li C, Yang H, Wang X, Tao H: The role of Th17/IL-17 in the pathogenesis of primary nephrotic syndrome in children. Kidney Blood Press Res 2013;37:332-345. 
3245 Kanai T, Shiraishi H, Yamagata T, Ito T, Odaka J, Saito T, Aoyagi J, Momoi MY: Th2 cells 325 predominate in idiopathic steroid-sensitive nephrotic syndrome. Clin Exp Nephrol $326 \quad 2010 ; 14: 578-583$.

3276 Zhu J, Yamane H, Paul WE: Differentiation of effector CD4 T cell populations ( ${ }^{*}$ ). Annu Rev $328 \quad$ Immunol 2010;28:445-489.

3297 Luo B, Feng L, Jintao D, Yafeng L, Shixi L, Nan Z, Bachert C: Immunopathology features of 330 chronic rhinosinusitis in high-altitude dwelling Tibetans. Allergy Rhinol (Providence) $331 \quad$ 2013;4:e69-76.

3328 Narozna B, Langwinski W, Szczepankiewicz A: Non-Coding RNAs in Pediatric Airway 333 Diseases. Genes (Basel) 2017;8:

3349 Zhu YJ, Mao D, Gao W, Hu H: Peripheral whole blood IncRNA expression analysis in 335 patients with eosinophilic asthma. Medicine (Baltimore) 2018;97:e9817.

33610 Zhang XY, Tang XY, Li N, Zhao LM, Guo YL, Li XS, Tian CJ, Cheng DJ, Chen ZC, Zhang LX: 337 GAS5 promotes airway smooth muscle cell proliferation in asthma via controlling miR338

11 Qiu YY, Wu Y, Lin MJ, Bian T, Xiao YL, Qin C: LncRNA-MEG3 functions as a competing 340

12 Li JQ, Hu SY, Wang ZY, Lin J, Jian S, Dong YC, Wu XF, Dai L, Cao L: Long non-coding RNA MEG3 inhibits microRNA-125a-5p expression and induces immune imbalance of Treg/Th17 in immune thrombocytopenic purpura. Biomed Pharmacother 2016;83:905-

13 Shao XS, Yang XQ, Zhao XD, Li Q, Xie YY, Wang XG, Wang M, Zhang W: The prevalence of Th17 cells and FOXP3 regulate T cells (Treg) in children with primary nephrotic syndrome. Pediatr Nephrol 2009;24:1683-1690.

14 Li YY, Wei SG, Zhao X, Jia YZ, Zhang YF, Sun SZ: Th17/Treg cell expression in children with 350 primary nephritic syndrome and the effects of ox-LDL on Th17/Treg cells. Genet Mol Res 2016;15: 
35215 Li Q, Wang Y, Li H, Shen G, Hu S: Ox-LDL influences peripheral Th17/Treg balance by modulating Treg apoptosis and Th17 proliferation in atherosclerotic cerebral infarction. Cell Physiol Biochem 2014;33:1849-1862.

16 Livak KJ, Schmittgen TD: Analysis of relative gene expression data using real-time

17 Yan L, Liu Z, Yin H, Guo Z, Luo Q: Silencing of MEG3 inhibited ox-LDL-induced inflammation and apoptosis in macrophages via modulation of the MEG3/miR-204/CDKN2A regulatory axis. Cell Biol Int 2019;43:409-420.

18 Liu MM, Wen L, Liu YJ, Cai Q, Li LT, Cai YM: Application of data mining methods to improve screening for the risk of early gastric cancer. BMC Med Inform Decis Mak 2018;18:121.

20 Sakaguchi S, Sakaguchi N, Asano M, Itoh M, Toda M: Immunologic self-tolerance 366

21 Chen W, Jin W, Hardegen N, Lei KJ, Li L, Marinos N, McGrady G, Wahl SM: Conversion of 370 peripheral CD4+CD25- naive T cells to CD4+CD25+ regulatory T cells by TGF-beta induction of transcription factor Foxp3. J Exp Med 2003;198:1875-1886.

37523 Bettelli E, Carrier Y, Gao W, Korn T, Strom TB, Oukka M, Weiner HL, Kuchroo VK: Reciprocal 376

24 Okada Y, Tsuzuki Y, Sato H, Narimatsu K, Hokari R, Kurihara C, Watanabe C, Tomita K, sulphate-induced colitis by promoting the up-regulation of macrophage-derived 
proinflammatory cytokines involved in T helper 17 cell polarization. Clin Exp Immunol 2013;174:459-471.

38325 Burkett PR, Meyer zu Horste G, Kuchroo VK: Pouring fuel on the fire: Th17 cells, the 384 environment, and autoimmunity. J Clin Invest 2015;125:2211-2219.

$385 \quad$ Figure legends

\section{Table 1}

387 Demographic and clinical characteristics of PNS patients and HC individuals.

$388 \quad$ Fig.1

389 The Treg/Th17 ratio was evidently decreased in PNS patients (* P value $<0.05$ vs. HC group)

390 A: The percentage of Th17 cells in the PNS group was significantly increased compared with that 391 in the HC group;

392 B: The percentage of Treg cells in the PNS group was markedly reduced compared with that in 393 the HC group.

$394 \quad$ Fig.2

395 Differential expression of MEG3, relevant miRNAs and relevant mRNAs in PBMC samples 396 collected from PNS and HC groups (* P value $<0.05$ vs. HC group)

397 A: PBMC MEG3 expression was increased in the PNS group compared with that in the HC group; B: PBMC miR-17 expression was inhibited in the PNS group compared with that in the HC group; C: PBMC RORyt mRNA expression was promoted in the PNS group compared with that in the HC 400 group;

401 D: The expression of PBMC miR-125a was inhibited in the PNS group compared with that in the 402 HC group;

403 E: The expression of PBMC FOXP3 mRNA was inhibited in the PNS group compared with that in 404 the HC group. 


\section{$405 \quad$ Fig.3}

406 Differential expression of MEG3, miR-17 and miR-125a in plasma samples collected from PNS and 407 HC groups (* P value $<0.05$ vs. HC group)

408 A: Plasma MEG3 expression was increased in the PNS group compared with that in the HC group;

409 B: Plasma miR-17 expression was inhibited in the PNS group compared with that in the HC group;

410 C: Plasma miR-125a expression was inhibited in the PNS group compared with that in the HC 411 group.

\section{$412 \quad$ Fig.4}

413 Pro-inflammatory cytokines were up-regulated in the PNS group (* $\mathrm{P}$ value $<0.05$ vs. HC group)

414 A: The level of IL-6 was highly increased in the PNS patients compared with that in the HC group;

415 B: The level of IL-1 $\beta$ was highly increased in the PNS patients compared with that in the HC group.

\section{$416 \quad$ Fig.5}

417 Ox-LDL regulated the expression of MEG3, relevant miRNAs and relevant mRNA in THP-1 cells (* 418 P value $<0.05$ vs. PBS group; ** $\mathrm{P}$ value $<0.05$ vs. ox-LDL+NC siRNA group)

419 A: The expression of MEG3 in the PBS group, ox-LDL+NC siRNA group and ox-LDL+MEG3 siRNA 420 group;

421 B: The expression of miR-17 in the PBS group, ox-LDL+NC siRNA group and ox-LDL+MEG3 siRNA 422 group;

423 C: The expression of RORyt mRNA in the PBS group, ox-LDL+NC siRNA group and ox-LDL+MEG3 424 siRNA group;

425 D: The expression of miR-125a in the PBS group, ox-LDL+NC siRNA group and ox-LDL+MEG3 siRNA 426 group;

427 E: The expression of FOXP3 mRNA in the PBS group, ox-LDL+NC siRNA group and ox-LDL+MEG3 428 siRNA group; 
429 F: The expression of RORyt protein in the PBS group, ox-LDL+NC siRNA group and ox-LDL+MEG3

430 siRNA group;

431 G: The expression of FOXP3 protein in the PBS group, ox-LDL+NC siRNA group and ox-LDL+MEG3

432 siRNA group.

$433 \quad$ Fig. 6

434 MEG3 regulated the expression of relevant miRNAs and mRNAs in THP-1 cells (* $\mathrm{P}$ value $<0.05$

435 vs. NC group)

436 A: The expression of MEG3 in THP-1 cells transfected with MEG3 compared with that in the 437 negative controls;

438 B: The expression of miR-17 in THP-1 cells transfected with MEG3 compared with that in the 439 negative controls;

440 C: The expression of RORyt mRNA in THP-1 cells transfected with MEG3 compared with that in 441 the negative controls;

442 D: The expression of miR-125a in THP-1 cells transfected with MEG3 compared with that in the 443 negative controls;

444 E: The expression of FOXP3 mRNA in THP-1 cells transfected with MEG3 compared with that in 445 the negative controls;

446 F: The expression of RORyt protein in THP-1 cells transfected with MEG3 compared with that in 447 the negative controls;

448 G: The expression of FOXP3 protein in THP-1 cells transfected with MEG3 compared with that in 449 the negative controls.

$450 \quad$ Fig.7

451 MEG3 could sponge miR-17 and miR-125a expression, while RORyt mRNA expression was 452 targeted by miR-17 (* P value $<0.05$ vs. blank group).

453 A: Bioinformatic tools and luciferase assay validated that MEG3 could sponge miR-17 expression; 
454 B: Bioinformatic tools and luciferase assay validated that RORpt mRNA was targeted by miR-17;

455 C: Bioinformatic tools and luciferase assay validated that MEG3 could sponge miR-125a 456 expression. 
Table 1. Demographic and clinical characteristics of PNS patients and HC individuals.

\begin{tabular}{lccc}
\multicolumn{1}{c}{ Characteristics } & HC (N=42) & PNS (N=40) & $P$ value \\
\hline Age (years) & $6.5 \pm 1.8$ & $6.1 \pm 2.2$ & 0.710 \\
Sex & & & 0.710 \\
$\quad$ Male & 30 & 26 & \\
$\quad$ Female & 12 & 14 & 0.538 \\
Histological classification & & & \\
$\quad$ Minor change & 1 & 0 & \\
Focal glomerulone & 2 & 1 & \\
MCNS & 7 & 8 & \\
MsPGN & 18 & 16 & \\
FSGS & 6 & 6 & \\
MN & 8 & 9 & \\
\hline
\end{tabular}



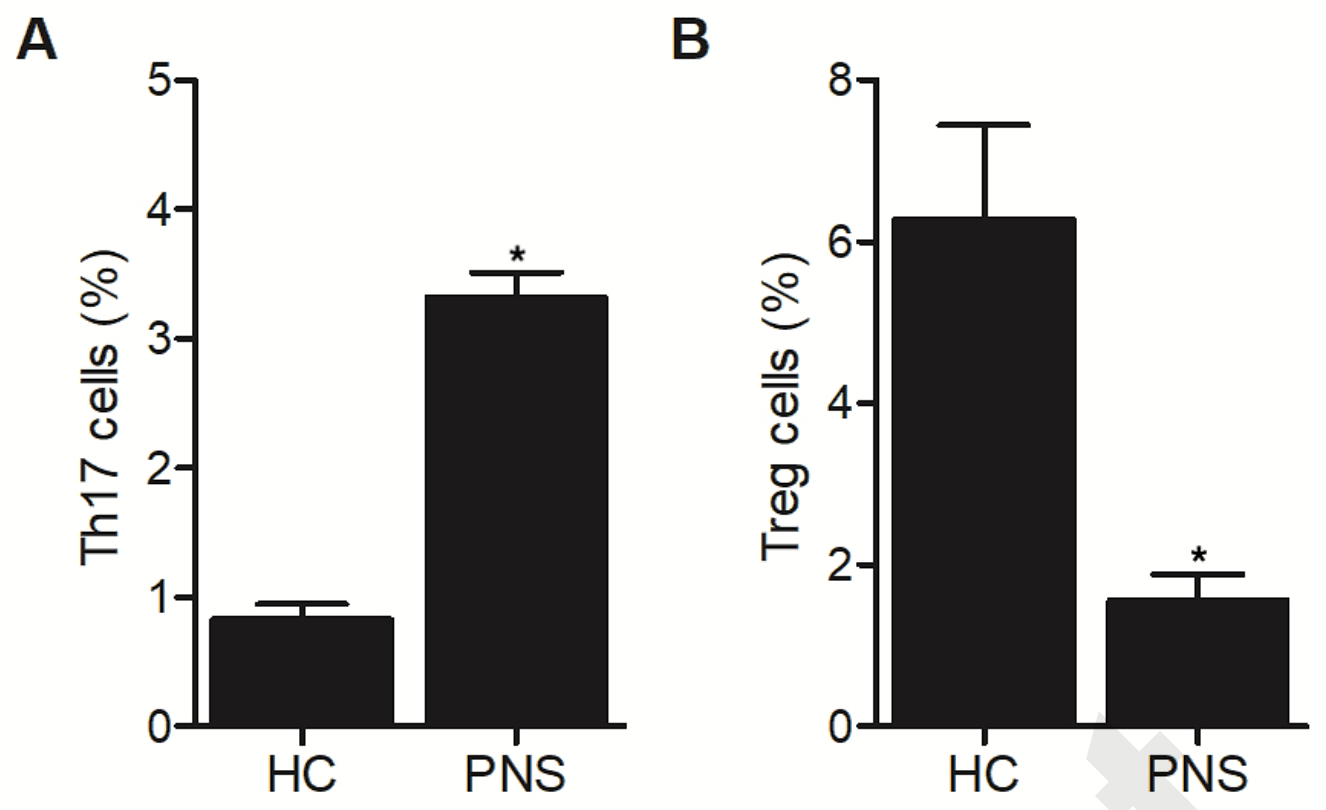

The Treg/Th17 ratio was evidently decreased in PNS patients ( ${ }^{*} \mathrm{P}$ value $<0.05$ vs. HC group)

A: The percentage of Th17 cells in the PNS group was significantly increased compared with that in the $\mathrm{HC}$ group;

$\mathrm{B}$ : The percentage of Treg cells in the PNS group was markedly reduced compared with that in the $\mathrm{HC}$ group. 

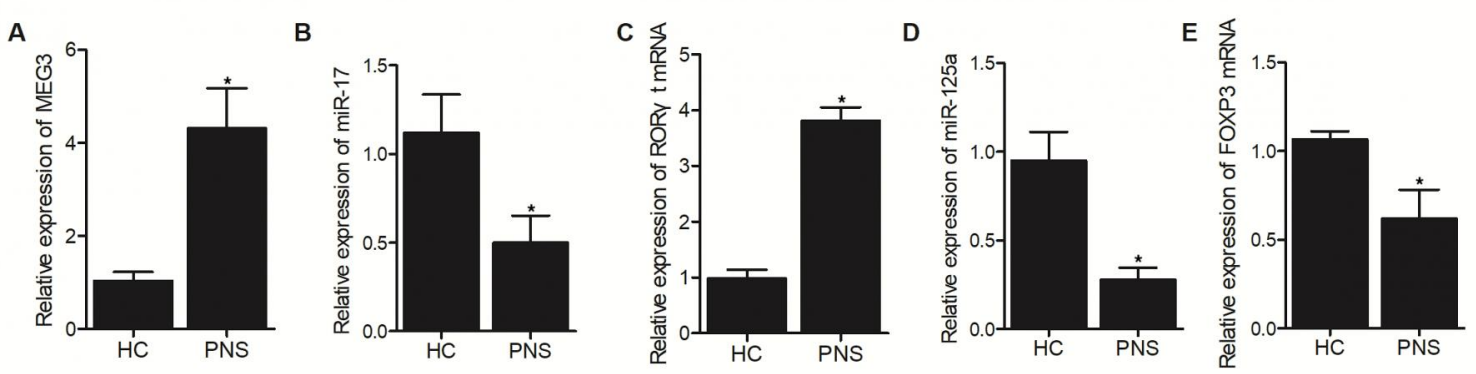

Differential expression of MEG3, relevant miRNAs and relevant mRNAs in PBMC samples collected from PNS and $\mathrm{HC}$ groups ( ${ }^{*} \mathrm{P}$ value $<0.05 \mathrm{vs}$. HC group)

A: PBMC MEG3 expression was increased in the PNS group compared with that in the HC group;

B: PBMC miR-17 expression was inhibited in the PNS group compared with that in the HC group;

C: PBMC RORyt mRNA expression was promoted in the PNS group compared with that in the $\mathrm{HC}$ group;

D: The expression of PBMC miR-125a was inhibited in the PNS group compared with that in the $\mathrm{HC}$ group;

$\mathrm{E}$ : The expression of PBMC FOXP3 mRNA was inhibited in the PNS group compared with that in the $\mathrm{HC}$ group. 
A

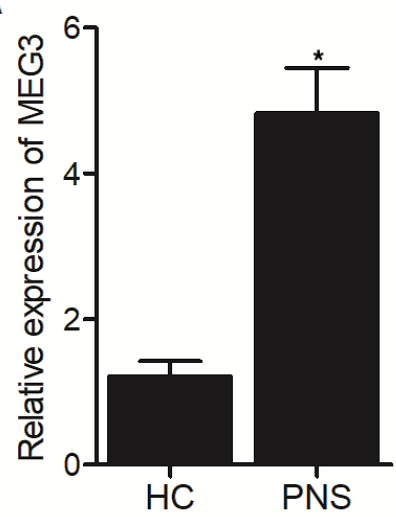

B

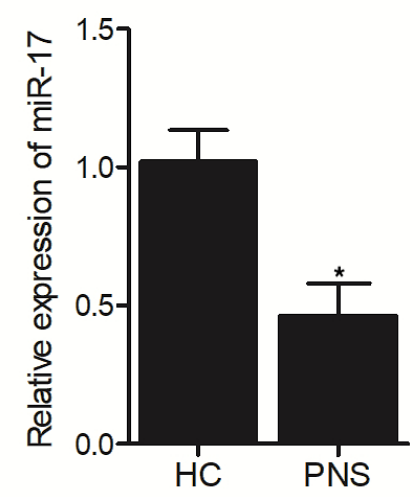

C

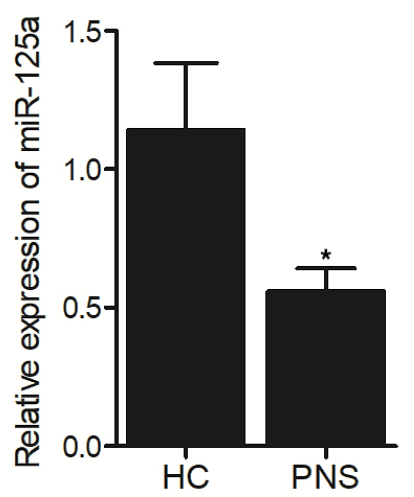

Differential expression of MEG3, miR-17 and miR-125a in plasma samples collected from PNS and HC groups ( ${ }^{*} \mathrm{P}$ value $<0.05$ vs. HC group)

A: Plasma MEG3 expression was increased in the PNS group compared with that in the HC group;

B: Plasma miR-17 expression was inhibited in the PNS group compared with that in the HC group;

C: Plasma miR-125a expression was inhibited in the PNS group compared with that in the HC group. 
A

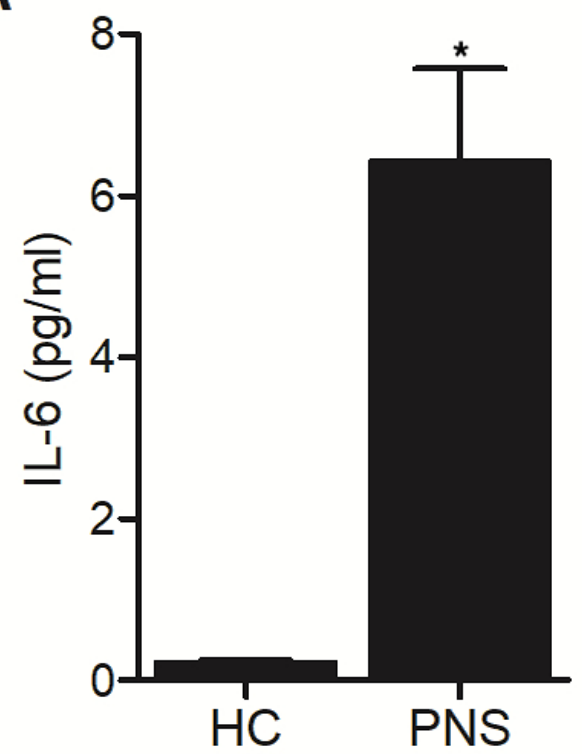

B

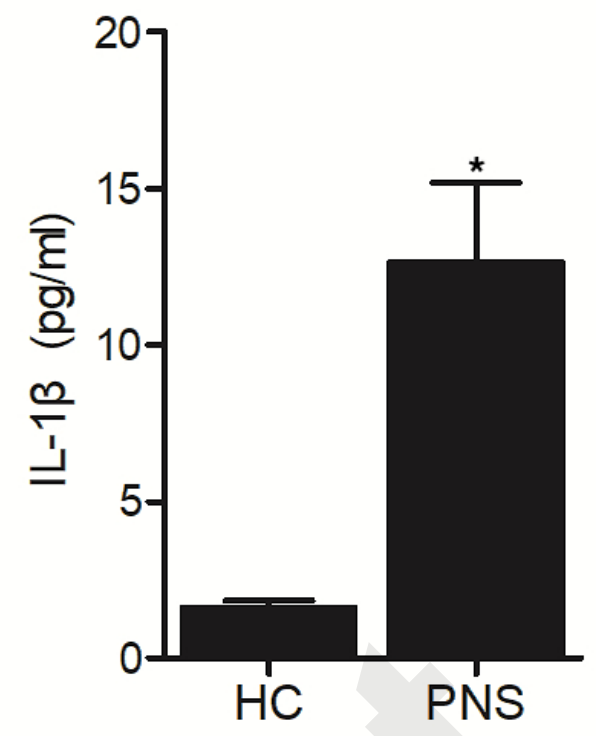

Pro-inflammatory cytokines were up-regulated in the PNS group $\left({ }^{*} \mathrm{P}\right.$ value $<0.05$ vs. HC group)

A: The level of IL-6 was highly increased in the PNS patients compared with that in the HC group;

B: The level of IL-1 $\beta$ was highly increased in the PNS patients compared with that in the HC group. 


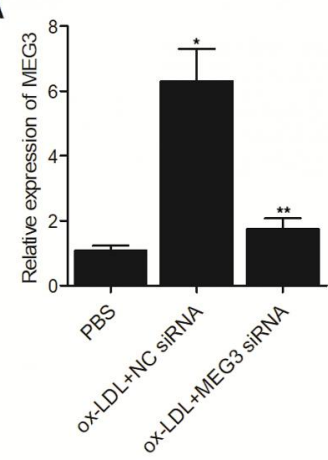

E

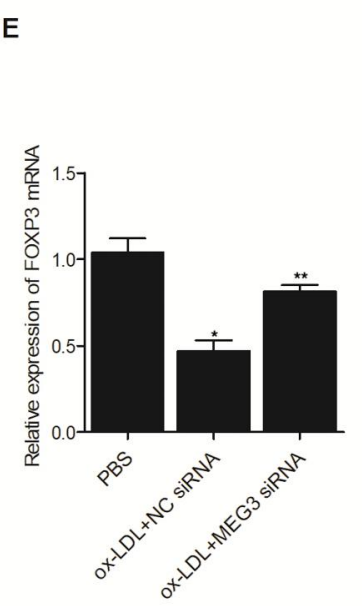

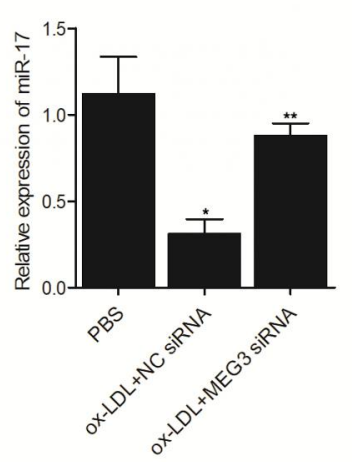
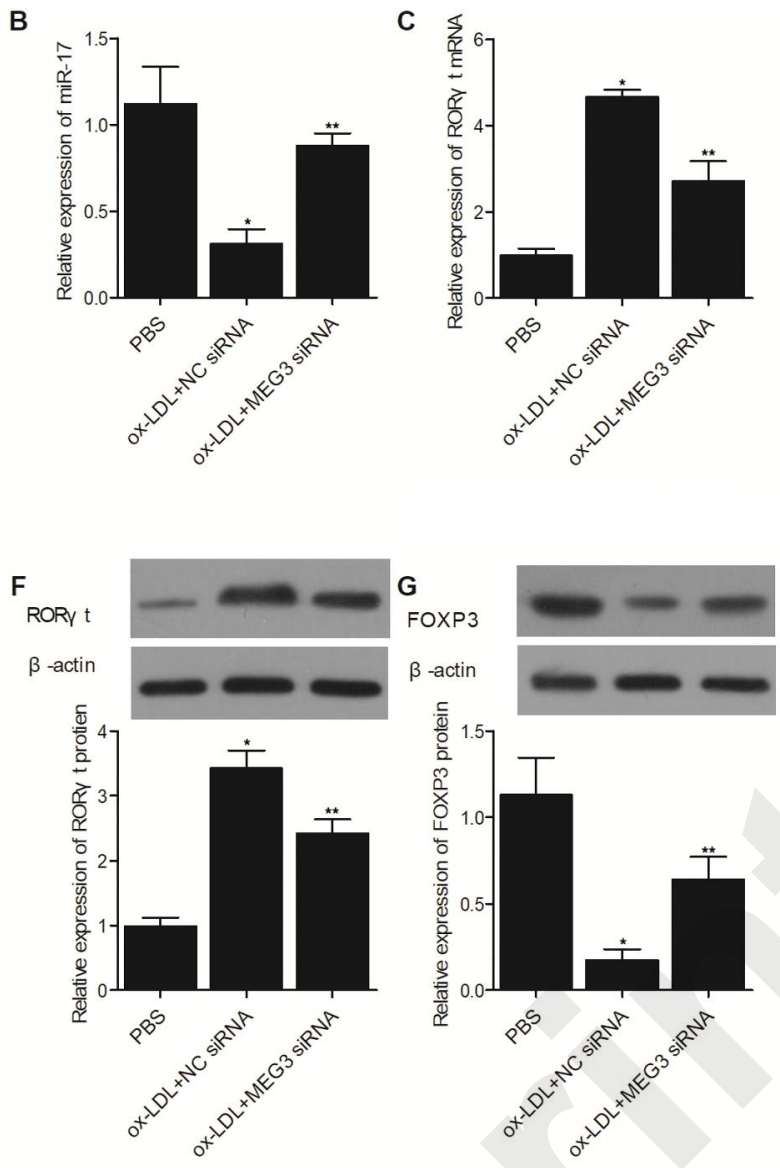

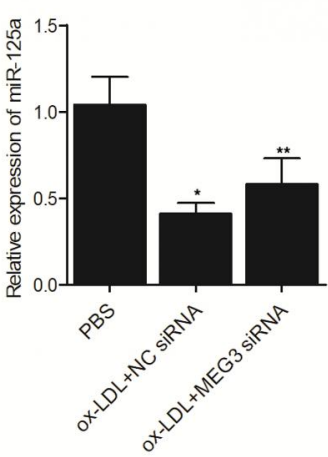

Fig.5

Ox-LDL regulated the expression of MEG3, relevant miRNAs and relevant mRNA in THP-1 cells ( ${ }^{*} P$ value $<0.05$ vs. PBS group; ${ }^{* *} P$ value $<0.05$ vs. ox-LDL+NC siRNA group)

A: The expression of MEG3 in the PBS group, ox-LDL+NC siRNA group and oxLDL+MEG3 siRNA group;

$B$ : The expression of miR-17 in the PBS group, ox-LDL+NC siRNA group and oxLDL+MEG3 siRNA group;

C: The expression of RORyt mRNA in the PBS group, ox-LDL+NC siRNA group and oxLDL+MEG3 siRNA group;

D: The expression of miR-125a in the PBS group, ox-LDL+NC siRNA group and oxLDL+MEG3 siRNA group;

$E$ : The expression of FOXP3 mRNA in the PBS group, ox-LDL+NC siRNA group and oxLDL+MEG3 siRNA group;

$\mathrm{F}$ : The expression of RORyt protein in the PBS group, ox-LDL+NC siRNA group and oxLDL+MEG3 siRNA group;

G: The expression of FOXP3 protein in the PBS group, ox-LDL+NC siRNA group and oxLDL+MEG3 siRNA group. 
A

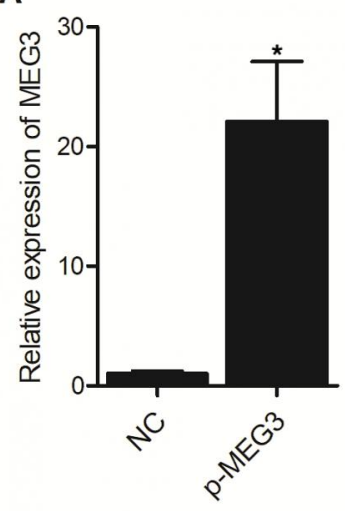

E

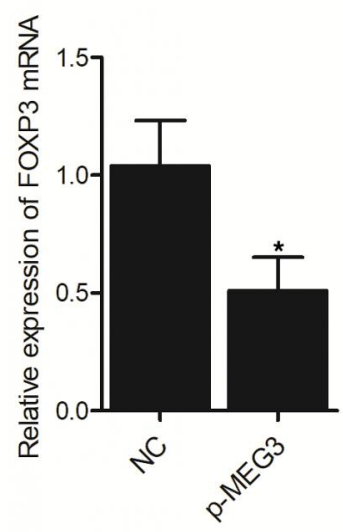

B

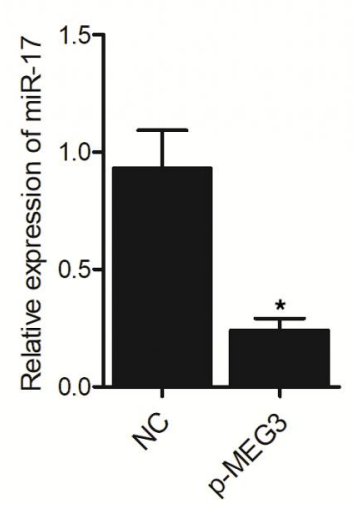

$\mathbf{F}$

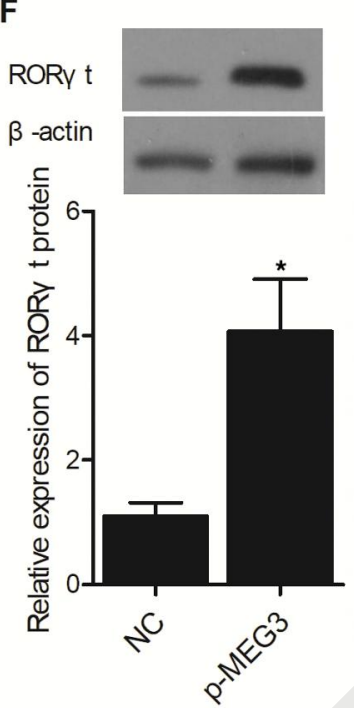

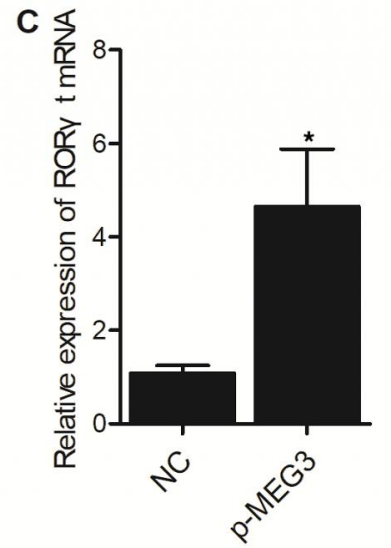

G
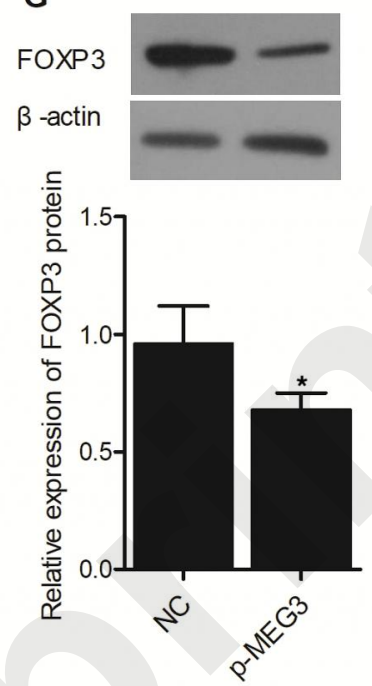

D

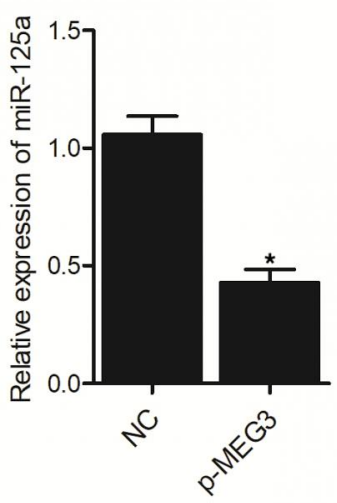

Fig.6

MEG3 regulated the expression of relevant miRNAs and mRNAs in THP-1 cells ( ${ }^{*} \mathrm{P}$ value $<$ 0.05 vs. NC group)

A: The expression of MEG3 in THP-1 cells transfected with MEG3 compared with that in the negative controls;

B: The expression of miR-17 in THP-1 cells transfected with MEG3 compared with that in the negative controls;

C: The expression of RORyt mRNA in THP-1 cells transfected with MEG3 compared with that in the negative controls;

D: The expression of miR-125a in THP-1 cells transfected with MEG3 compared with that in the negative controls;

E: The expression of FOXP3 mRNA in THP-1 cells transfected with MEG3 compared with that in the negative controls;

F: The expression of RORyt protein in THP-1 cells transfected with MEG3 compared with that in the negative controls;

G: The expression of FOXP3 protein in THP-1 cells transfected with MEG3 compared with that in the negative controls. 
A

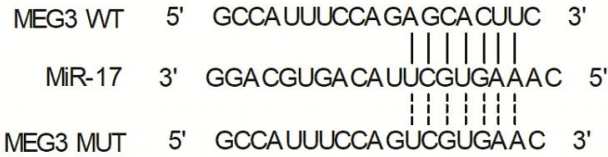

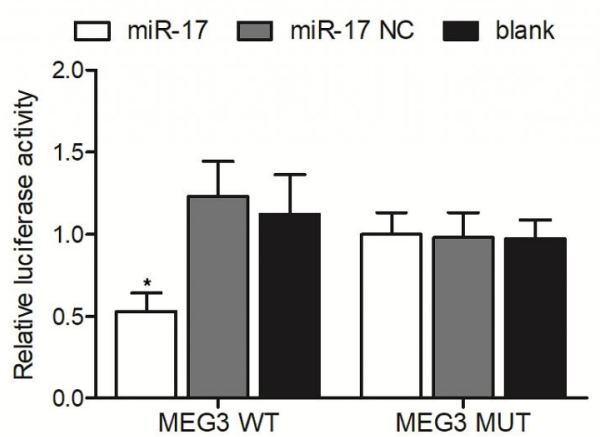

C

MEG3 WT 5' ACCCGACCACGUCU CAgGA 3'

MiR-125a 3' GUCCAAUUUCCCAGAGUCCC 5'

MEG3 MUT 5' ACCCGACCACCÁGÁGUCCA $3^{\prime}$

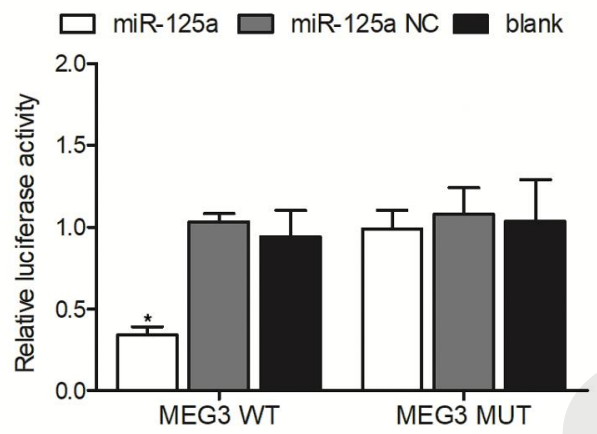

B

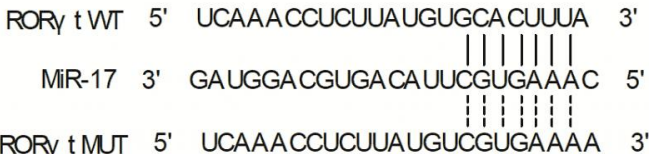

RORY tMUT 5' UCAAACCUCUUAUGUCGUGAAAA 3

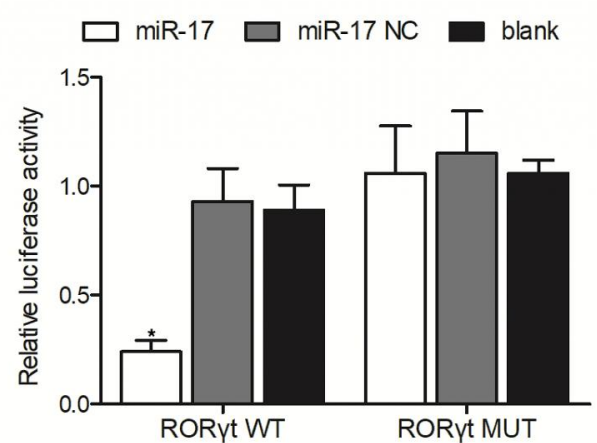

MEG3 could sponge miR-17 and miR-125a expression, while RORyt mRNA expression was targeted by miR-17 ( ${ }^{*} \mathrm{P}$ value $<0.05$ vs. blank group).

A: Bioinformatic tools and luciferase assay validated that MEG3 could sponge miR-17 expression;

B: Bioinformatic tools and luciferase assay validated that RORyt mRNA was targeted by miR-17;

C: Bioinformatic tools and luciferase assay validated that MEG3 could sponge miR-125a expression. 